\title{
COLOR IMAGE RETRIEVAL USING TAKEN IMAGES
}

\author{
S. Selvam \\ Bharathiar University, Tamilnadu - INDIA
}

\begin{abstract}
Now a days in many applications content based image retrieval from large resources has become an area of wide interest. In this paper we present a color-based image retrieval system that uses color and texture as visual features to describe the content of an image region. To speed up retrieval and similarity computation, the database images are segmented and the extracted regions are clustered according to their feature vectors. This process is performed offline before query processing, therefore to answer a query our system need not search the entire database images; instead just a number of candidate images are required to be searched for image similarity. Our proposed system has the advantage of increasing the retrieval accuracy and decreasing the retrieval time. The experimental evaluation of the system is based on a 1,000 real taken color image database. From the experimental results, it is evident that our system performs significantly better and faster compared with other existing systems. In our analysis, we provide a comparison between retrieval results based on relevancy for the given ten classes. The results demonstrate that each type of feature is effective for a particular type of images according to its semantic contents, and using a combination of them gives better retrieval results for almost all semantic classes.
\end{abstract}

Key words: $\quad$ CBIR, HARP, Recall, Precision, BBF, LSF

\section{Introduction (Heading 1)}

CBIR utilizes representations of features that are automatically extracted from the images themselves. Most of the current CBIR systems allow for querying-by-example, a technique where in an image or part of an image is selected by the user as the query. The system extracts the features of the query image, searches through the database for images with similar features, and displays relevant images to the user in order of similarity to the query.

CBIR systems attempt to exploit the visual information inherent in images, thus providing a more realistic perceptual representation of an image. In this context, content includes among other features, perceptual properties such as color, texture, shape, and spatial relationships. Many CBIR systems have been developed that analyze, compare and retrieve images based on one or more of these features. Some systems have achieved various degrees of success by combining both content-based and text-based retrieval.

\section{The New System}

Our new system fusions the process of training image input, feature extraction, genetic optimization, clustering and similarity matching. Here the query result is better than any other existing systems. The main objective of this paper is to build more generalized CBIR system which increase the searching ability and provide more accurate results. To improve the retrieval accuracy the system has taken the feedback from the user automatically. The main aim of this new system is to minimize the computation time and user interaction. But in our newly constructed system, the time taken for analysis is meager, because it passes through various processing stages based on the user's threshold values. The step of this study is to reduce the gap between high and low level features as CBIR calculates the similarity between user query and repositories image. It may lead to unwanted retrieval of images. By using HARP, it groups the output images and a representative image from every cluster. The subsequent process is performance evaluation on the basis of speed and accuracy, because it gives strong impact on the implementation.

Training image input: The image inputs are trained with different weights. The training part outputs the classifying result and stores it in the feature database. All these steps performed offline and each class will be indexing along with its associated classID.

Feature Extraction: There are various kinds of visual features to represent an image, such as color, texture, shape, and spatial relationship. Since one type of features can only represent part of the image properties, a lot of work done on the combination of these features. The feature of each image is very much smaller in size compared to the image data, so the 


\section{Yinternational Research Journal}

p-ISSN 2202-2821 e-ISSN 1839-6518 (Australian ISSN Agency)

feature database contains an abstraction of the images in the image database.

Genetic optimization: It is used to find solution for complicated problems. It is based on heuristic approach that imitates the process of natural selection. It is used exclusively for the purpose of optimization. Each intermediary solution can be mutated and changed.

HARP - a Clustering algorithm: The algorithm is based on bottom-up approach. Initially pick up each element among all current cluster on the basis of smallest distance by merging all the selected and related data on various clusters. In HARP algorithm, the accuracy level of clustering is more by using relevance indexing and merge score. The scalability level is also very high. The time taken for finding the closest cluster is very less.

Similarity Matching: Using the similarity metrics defined for color, texture and shape, the similarity distances between the query image and the centroid image of each class are calculated. The smallest distance (most similar) will determine to which the image belongs. The class with the smallest distance is returned and the images in this class will be compared with the query image.

The main aim of this research paper is to determine which images are the most similar one to any given query image. The new proposed method is used to combine the features of structure and block-based to get the accuracy in an increased level. Image matching is performed by comparing feature histograms out of a feature database. We only need to compute the query image's feature histogram and perform a histogram comparison over the whole database. For the current application we used the intersection similarity measure. Here the data-set for ten classes retrieved and checked its similarity in-terms of recall from the taken image database. For this purpose we have taken 100 (projected to 1,000) image sets as sampling to increase the accuracy.

Here we synthesis the three features results in a robust manner. After extracting and normalizing the image feature vectors, we need to determine which images the most similar ones to any are given query image. The matching of image is performed by comparing the robustness value. We only need to compute the query image and perform a comparison over the whole database. For the current application we used the intersection similarity measure. By averaging the results for each class we obtain a qualitative performance analysis for all image classes.

Here we have calculated the recall by the measurement relevancy. By calculating the hit ratio we can easily identify the performance of the method. If the hit ratio is greater, the
Vol. 7 No. 12017

82800701201702

performance of the method is also high. From the hit ratio, we calculate the precision, accuracy, sensitivity, specificity and Fvalue. We believe that a sophisticated feature selection method might improve the results of our new method by applying larger weights to the line segment features.

Our new method clearly proves that it is superior.

For the current experiments, we have used our own taken real images, viz ThaSel. The task is to find similarity among various classes of the taken images. Due to computational resource and time limitations we provide a less comprehensive representation of the experiments performed with the dataset containing 1,000 images. The results are evaluated with precision recall graphs and compared with two well-known methods, namely global color histograms and the local invariant feature histogram algorithm [59].

Since color histograms are commonly used in CBIR, they serve well as a comparative feature. However, in our experiments we have obtained a 32-bit color-histogram from the $\mathrm{YCbCr}$ chrominance channel of each image. The invariant feature histogram method is adopted due to its good performance as a local texture-based feature. The method constructs invariant features by applying nonlinear kernel functions to color images and integration over all possible preprocessing activities like Gaussian blur, random noise, affine, sparkle light, projection and rotation of image by 90 degrees counter clock wise.

\section{Retrieval Results}

For the retrieval task we use the global structure-based features Hglobal. For the current experiment we are interested in the performance of global structure features and if they are suitable to be combined with local descriptors. Therefore, we combine the global structure features with block-based features to obtain the greater accuracy. The local method (BBF) computes pixel value distributions of equally sized blocks.

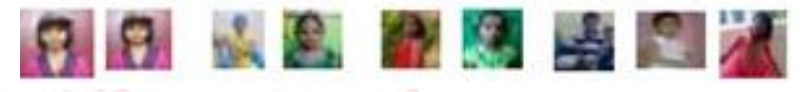

Sample image retrieval result for an image out of the class: Baby

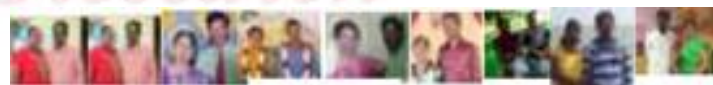

Sample image retrieval result for an image out of the class: Couple

Figure 1: Sample image retrieval result obtained from 100 image collection 


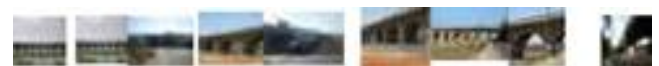

Sample image retrieval result for an image out of the class: Bridge

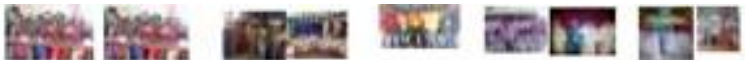

Sample image retrieval result for an image out of the class: Group

Figure 2: Sample image retrieval result obtained from 100 image collection
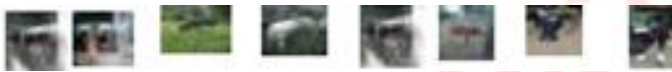

Sample image retrieval result for an image out of the class: Cow

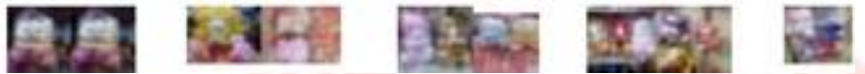

Sample image retrieval result for an image out of the class: Doll

Figure 3: Sample image retrieval result obtained from 100 image collection
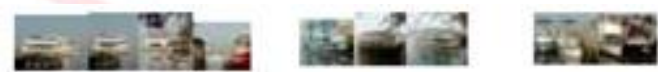

Sample image retrieval result for an image out of the class: Boat

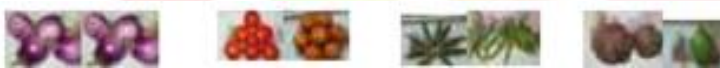

Sample image retrieval result for an image out of the class: Vegetable

Figure 4: Sample image retrieval result obtained from 100 image collection
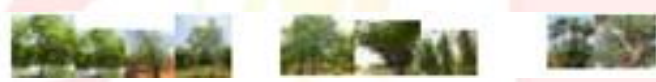

Sample image retrieval result for an image out of the class: Tree
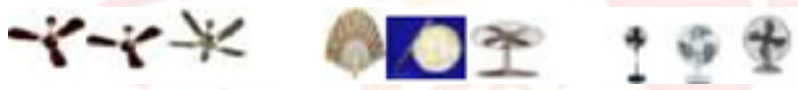

Sample image retrieval result for an image out of the class: Fan

Figure 5: Sample image retrieval result obtained from 100 image collection

The synthesis of the taken image features results in a robust semantic image descriptor. After extracting and normalizing the image feature vectors, we need to determine which images the most similar ones to any are given query image. Image matching is performed by comparing feature histograms out of a feature database, where the feature histogram of each image is computed offline. We only need to compute the query image's feature histogram and perform a histogram comparison. For the current application we used the robust intersection similarity measure and clustering technique.

In our evaluation strategy, we use each image from the taken image data set as the query image to compute the precision and recall. By averaging the results for each class we obtain a qualitative performance analysis for all image classes.
We selected some sample queries from the datasets and display their results in Figure 1to Figure 5, which were obtained from the 100 image data-set (projected for 1,000 dataset). Each panel displays sample results, where the top-left image is the query image. All other images are ranked in decreasing similarities, where the number above each image points out the similarity, with values in the range of $[0,1]$, with one denoting an identical match with the query image. The result in Figure 1 to 5 reveals that the first eight retrieved images belong to the query image class.

Figure 6 shows the average recall versus the number of retrieved images graph for all ten classes of the 100 image database (projected for 1,000 dataset). Each graph in the figure shows five different curves representing the performance of the line segment features, block-based features, invariant feature histogram, color histogram and our new method (the combination of the local and global based features). It can be seen that our new method i.e. combination of the structure and block-based features performs best for most of the classes (eight out of ten), for the first 100 retrieved images.

For fan class the line segment feature and for doll class the color histogram method is best and for all other classes our new method is superior to any other methods.

For baby class our new method acts as a median between line segment feature and block based feature. For couple class, our method and block based feature possess on a same rail. For bridge class the recall value of block based feature is increased in a constant manner. The third point is increasing in an abnormal state. For group class and cow class color histogram moved in a constant way and there is an alternate up and down for block based feature. For doll class invariant feature histogram moves in a constant manner. For boat class the recall value of $\mathrm{BBF}$ is very near to our method. For vegetable class our method is coincided with BBF.

For class fan and doll classes use global line structure feature performs better than the combination with the block-based feature (BBF). This can be explained with a very prominent geometric structure of the fan and the doll, which is here best encoded by the global line structure features.

We believe that a sophisticated feature selection method might improve the results of the combined features by applying larger weights to the line segment features. However, for the current experiments we did not implement feature selecting, feature weighting or any further relevance feedback methods to improve the results. 


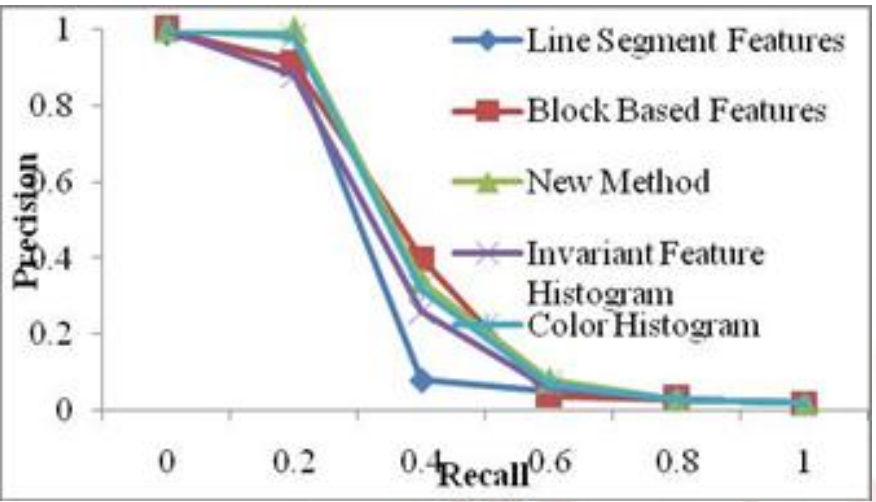

Figure 6: Precision-recall graph for the 100 image data-set, where the graph is averaged over all images and classes representing an overall performance measure.

In the class cow our features perform worse than the global color-histogram. A possible reason for that might be found in the color distribution of the image class, i.e. about $80 \%$ of cows in that class are standing or running, which introduce a significant background. In terms of pixels it clearly shows that color dominates the information. The fraction of pixels belonging to cow is much smaller than the background area and thus, color is a stronger feature than structure.

Though the curves of some classes look quite similar to each other, the overall precision recall graph in Figure 6 and Figure 7 proves the superior performance of our features.

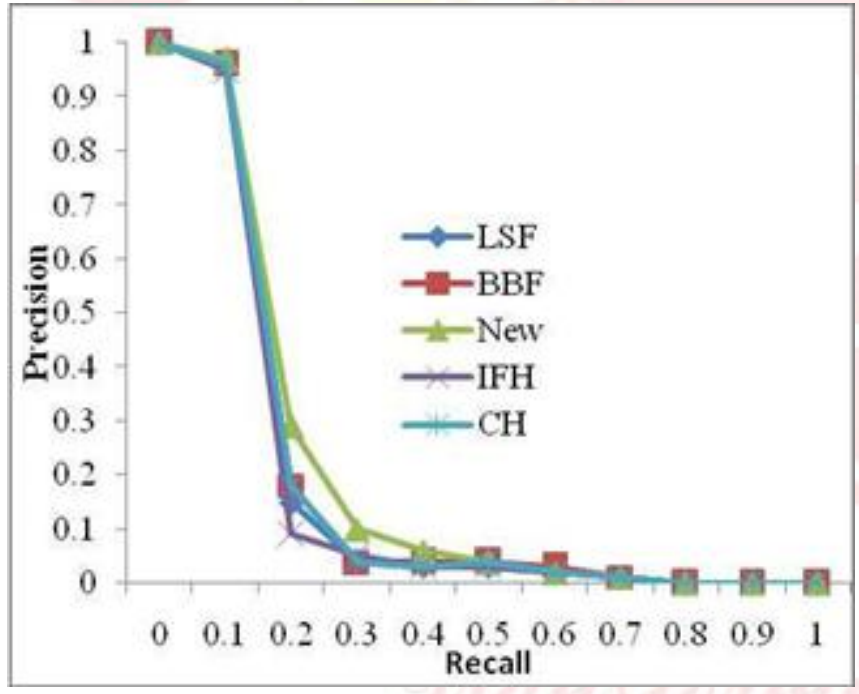

Figure 7: Precision-recall graph for the 1,000 image database (projected), here the graph is averaged over all images \& classes representing an overall performance measure

The graph represents the averaged precision-recall over all ten classes. The figure shows that our new method reaches a precision of $100 \%$ until a recall of about $20 \%$ is reached. An interesting observation can be found in the part between a recall of about 0.6 and 0.9 , where the color-histogram performs better than our new method. Our investigations revealed that the higher performance of the color-histogram for the class cow resulted in a superior recall for this part of the curve.

Figure 7 depicts the precision-recall graph averaged for the larger dataset (projected to 1,000 images). As well as for the first data-set, we compute the precision recall curve for all other features. Our new method performs better than the others methods.

\section{Invariance Analysis}

The results shown so far give a good idea of the proposed new method feature's performance. We give a measure of the robustness or invariance for all methods. It is of greater importance for CBIR applications to be invariant or robust under taken image activities with some basic transformations like changes of the brightness and rotation.

Hence we conduct an invariance analysis, where we compute all features for each image for two newly created data-sets. To cope with these kinds of basic transformations, we have derived a data-set from taken 1,000 image database. For the actual invariance test we determine the similarity between each feature of the database. We take the measurement of robustness to compute the similarity from the basic transformed image data-set.

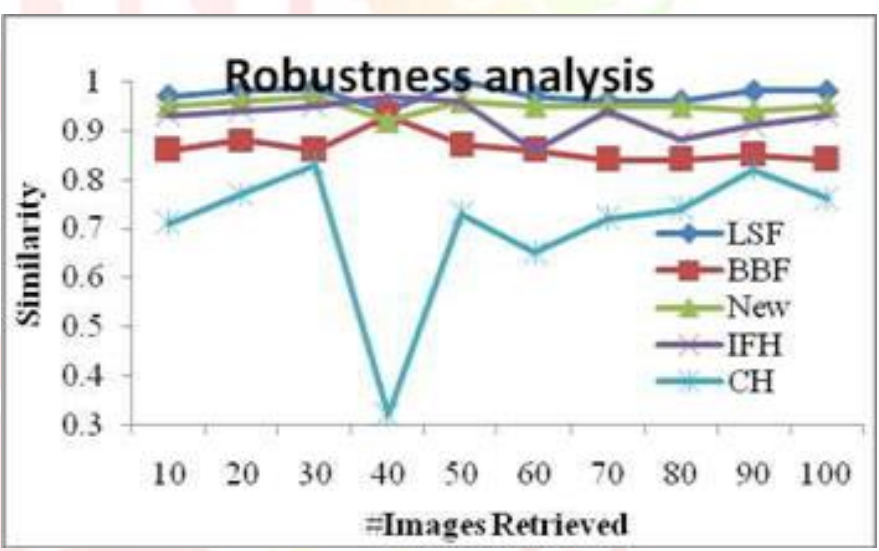

Figure 8: Robustness analysis of the 1,000 image database for different brightness.

The overall similarity levels for all methods are robust except no. of images 40 for color histogram method. As measure to compare the original image datasets and the transformed image we use the L1-Norm, resulting in a value between 0 and 1,1 indicating $100 \%$ invariance. The results are displayed in Figure9, where we can see five similarity versus number of image retrieved. The curves show the variation of the features taken from the original image data and the data-set with a different transforms. From the figure we can conclude that the line segment feature performs best and our new method features second best and the color histogram the worst. Our 
new method features is slightly worse than the line segment features. All features are equally robust to transformation.

The LSF have clear invariance properties that constitute their usefulness to CBIR applications. Moreover in our new method we have combined the global structure-based features with block based features. The block-based features are derived from equally sized non-overlapping 4 X 4 image blocks. Although the LSF produce good results, our new method improves the performance. The precision recall graphs, averaged over the whole image database, reveal for both image data-sets in our method perform better than any other method. It has to be said, that for some classes the features perform slightly worse, but averaged over all classes the precision recall graphs document a better performance.

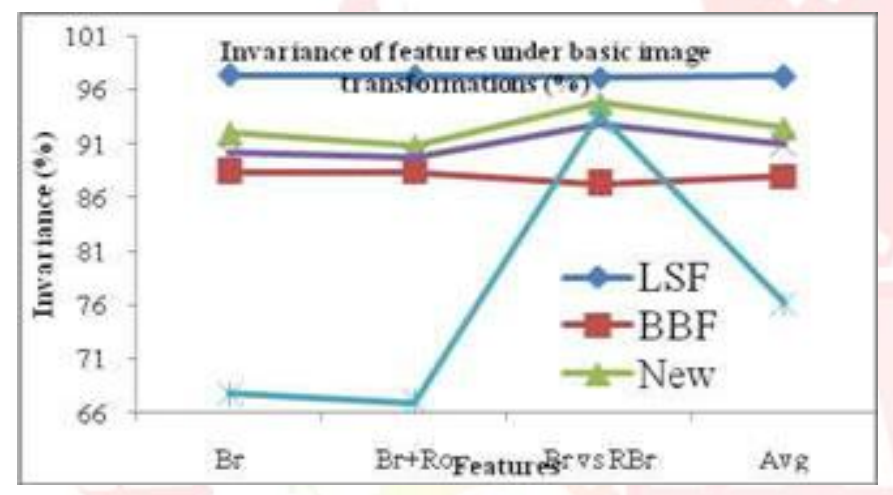

Figure 9: Averaged feature invariance representation.

The feature robustness analysis of the experiments revealed, that the LSF perform best in average, under changes of the brightness and under changes of the brightness plus rotation with a mean invariance of the feature of $97.23 \%$.

The invariance of our new method is with $92.48 \%$ slightly worse than the very robust LSB method with $97.23 \%$ invariance. Although the block-based features perform, according to the precision recall graphs more often better than the color histogram method, their combination gains in performance and robustness towards image transformations. The obtained results encourage for further enhancement, i.e. feature selection and/or sophisticated weighting schemes that might improve the feature combination process, leading to better results.

The mean of all the said classes are accepted at 30\% level of significance. The recall value of baby and doll is good. Our new method is suited for more than $70 \%$ of the classes. Hence it is proved that our new method is superb. The hit ratios of all the classes (except bridge and fan) have been accepted at $15 \%$ level of significance. The accuracy of all the classes has been accepted at $10 \%$ level of significance. The Precision and Specificity of all the classes are strictly intersected in a same manner. The sensitivity of all the classes is accepted at $30 \%$ level of significance. The F-measure of all the classes is accepted at $15 \%$ level of significance. $90 \%$ of the statistical methods supported our new method with respect to taken images.

\section{Conclusion}

The explosive growth of image data leads to the need of research and development of Image Retrieval. CBIR is currently a keen area of research in the field of multimedia databases. Various research works had been undertaken in the past decade to design efficient image retrieval techniques from the image databases. More précised retrieval techniques are needed to access the large image archives being generated, for finding relatively similar images. In this work the GA is combined with HARP clustering algorithm to improve the retrieval accuracy of the system. Getting lower computational time and retrieving relevant and accurate image is possible by using CBIR. In future we have a proposal to disseminate the features selections and use other distance measures to improve the overall results.

The efficiency of the new system is improved by considering candidate images for similarity computation purpose i.e. not considering the whole database images. A candidate image lies in the same cluster with the query image the benefit of the clustering process clearly proved the retrieval accuracy.

\section{References}

[1]. Thomas S. Huang, Yong Rui, and Shinh-Fu Chang, Image retrieval: Past, Present and Future, International Symposium on Multimedia Information Processing, 2015.

[2]. C. C. Venters and M. Cooper, Content-based image retrieval, Technical Report, JISC Technology Application Program, 2014.

[3]. M. Myron Flickner, H. Sawhney, W. Niblack, J. Ashley, "Query by image content: The QBIC system”, In IEEE Computer, pp. 23-31, Sept.2015.

[4]. Chad Carson, Serge Belongie, Hayit Greenspan, Jitendra Malik, "Blobworld: Image segmentation using Expectation-Maximization and its application to image querying", Third International Conference on Visual InformationSystems, 2015. In Proceedings of IEEE International Conference on Image Processing, pp.568-571,2015 [11]

[5]. Selvam, S., \& S. Thabasu Kannan. " Analysis of the major issues in Cloud Computing Environments." IARS' International Research Journal [Online], 5.2 (2015) 
[6]. V. E. Ogle and M. Stonebraker, Chabot: retrieval from object oriented data base of images, IEEE Computer, vol. 28, no. 9, pp. 40-8, Sept. 2015.

[7]. Selvam, S. and S. Thabasu Kannan, "Image Retrieval Optimization With Genitic Algorithm", published IJAER, International Journal of Applied Engineering Research as Special issue, Volume No 10, Issue No 55(2015) and IJAER is indexed by SCOPUS, And also listed in Anna University Chennai Annexure II-2014(Sl.No.8565).

[8]. Michael Ortega, Yong Rui, Kaushik Chakrabarti, Sharad Mehrotra and Thomas S. Huang, Supporting Similarity Queries in MARS. In Proceeding of the ACM International Multimedia Conference, pp. 403-413, 2014.

[9]. Tat-Seng Chua, Wai-Chee Low, and Chun-Xin Chu, Relevance feedback techniques for color-based image retrieval, In Proceedings of Multi-Media Modeling'13, IEEE Computer Society, pp 24-31, 2013.

[10]. SELVAM, S.; KANNAN, S. Thabasu. An Integration of Genetic Algorithm and Projected Clustering for Optimization of Content Based Image Retrieval System. IARS' International Research Journal, [S.1.], v. 4, n. 2, aug. 2014. ISSN 1839-6518.

[11]. D. Feng, W. Siu, H. Zhang (Eds.), "Color Image Retrieval and Management. Technological Fundamentals and Applications," Multimedia Signal Processing Book, Chapter1, Springer-Verlag, Berlin Heidelberg New York, 2013, page:1-26.

[12]. Selvam S. and S. Thabasu Kannan, "A New Technique for Color-Based Image Retrieval System Using Histogram", published IJAER, International Journal of Applied Engineering Research as Special issue, Volume No 10, Issue No 82(2015), ISSN 0973-4562 and IJAER is indexed by SCOPUS.

[13]. J. Smithand S. Chang, "Visualseek: A Fully Automated Content-Based Image Query System," Proceedings of the 4th ACM international conference on Multimedia table of contents, Boston, Massachusetts, United States, Nov.2016, pp.87-98.

[14]. SELVAM, S.; KANNAN, S. Thabasu. Analysis of the major issues in Cloud Computing Environments. IARS' International Research Journal, [S.1.], v. 5, n. 2, aug. 2015. ISSN 1839-6518.

[15]. J. Caicedo, F. Gonzalez, E. Romero, E. Triana, "Design of an Image Database with Content-Based Retrieval Capabilities, "In Proceedings of the 2nd Pacific Rim conference on Advances in image and video technology,Santiago,Chile,December1719,2014.

[16]. R. Zhang, and Z. Zhang, "A Clustering Based Approach to Efficient Image Retrieval, "Proceedings of the 14th IEEE International Conference on Tools with Artificial Intelligence (ICTAI'14), Washington,DC,Nov.2014,pp.339-346.

[17]. A. Natsev, R. Rastogi, and K. Shim, "WALRUS: A Similarity Retrieval Algorithm for Image Databases," IEEE Trans. On Knowledge and Data Engineering, vol.16, pp. 301-318, Mar. 2014.

[18]. Selvam S. and S. Thabasu Kannan, “An Empirical Review on Enhancing the Robustness of Multi resolution Water Marking", published IJAER, International Journal of Applied Engineering Research as Special issue, Volume No 10, Issue No 82(2015), ISSN 0973-4562 and IJAER is indexed by SCOPUS.

[19]. R. Picardand and T. Kabir, "Finding Similar Patterns in Large Image Databases," Proc. IEEE Int. Conf. Acoustics, Speech and Signal Processing, vol.5, pp. 161-164, NewYork2015.

[20]. D. Zhang, "Improving Image Retrieval Performance by Using Both Color and Texture Features," In Proc. of IEEE 3rd International Conference on Image and Graphics (ICIG14), Hong Kong, China, Dec.18-20,2014,pp.172-175.

[21]. R. Datta, J. Li, and J. Wang, "Content-based image retrieval-approaches and trends of the new age," ACM Computing Surveys, vol.40, no.2, Article5, pp.1-60, April2015. 